\title{
SATURATION BEHAVIOR OF EXTRINSIC PHOTOCONDUCTIVITY IN GaP LIGHT EMITTING DIODES AT HIGH INFRARED INTENSITIES
}

\author{
K. Moser and W. Prettl \\ Institut für Angewandte Physik, Universität Regensburg \\ 8400 Regensburg, West Germany
}

Received October 1, 1985

Saturation of extrinsic photoconductivity in GaP:N( $\mathrm{Zn}, \mathrm{Te}$ ) diodes could be achieved by excitation with a $\mathrm{TEA}^{-\mathrm{CO}_{2}-}$ laser. At wavelengths in the $10 \mu \mathrm{m}$ range intensities of several $100 \mathrm{~kW} / \mathrm{cm}^{2}$ being near the damage threshold were applied. Carrier 1 ifetimes of 60 ps at $4.2 \mathrm{~K}$ and 200 ps at $77 \mathrm{~K}$ could be estimated. The only conceivable mechanism explaining these short time constants is the capture of infrared excited holes by ionized shallow acceptors in the highly compensated p-side of the diode.

Key words: GaP light emitting diodes, ir-detection, saturation, response time

\section{Introduction}

Extrinsic photoconductivity and infrared excited electroluminescence have been observed in GaP light emitting diodes (LEDs) [1,2] providing a simple method of infrared to visible up-conversion. At low temperatures neutral shallow impurities are ionized by infrared radiation of suitable quantum energy and electrons and holes drift under the effect of an electric forward bias field into the pn-junction yielding visible luminescence by radiative interband recombination. In contrast to other up-conversion schemes involving laser pump sources, in the LED up-converter the deficient energy of visible photons 
is simply supplied by the bias voltage source.

By transient infrared excitation spectroscopy applying short $\mathrm{CO}_{2}$ laser pulses the detection time constants were evaluated to be smaller than 0.5 ns and 2 ns for photoconductivity and up-conversion, respectively [3,4]. These upper limits were determined by the duration of the laser pulses and the finite bandwidth of the detection device. Investigation of the evolution in time of the infrared induced electroluminescence spectrum showed that the radiative transitions were due to the well known donor-acceptor and nitrogen pair recombinations and in addition due to free hole-to-bound-donor transitions not yet observed before in GaP at Iiquid helium temperature [5]. This 1atter process turned out be very fast being responsible for the observed time constant in up-conversion.

In this paper we report on the saturation behaviour of photoconductivity under high intensity irradiation by a pulsed $\mathrm{CO}_{2}$ laser. Saturation intensities as high as $670 \mathrm{~kW} / \mathrm{cm}^{2}$ and $180 \mathrm{~kW} / \mathrm{cm}^{2}$ for $4.2 \mathrm{~K}$ and $77 \mathrm{~K}$, respectively, were derived from the measurements indicating time constants much shorter than those observed by transient excitation.

\section{Experimenta1}

The measurements were carried out on green emitting GaP:N(Zn,Te) LEDs without the infrared absorbing plastic cover. The diodes were prepared by liquid phase epitaxy on Te-doped n-conducting substrates. Alloyed metal electrodes covered the full size of the substrate and had the shape of a narrow cross on the opposite face leaving sufficient space for ir-irradiation into the p-side of the diodes. The samples were mounted in an optical cryostat and immersed in 1 iquid helium or liquid nitrogen. A TEA-CO 2 laser at $10.6 \mu \mathrm{m}$ wavelength emitting a strongly modulated pulse train due to mode beating was employed as an exciting source $[6,7]$. The photoconductive signal of single laser shots measured across a $50 \Omega$ load resistor was recorded using a Tektronix $\mathrm{R} 7912$ transient digitizer. A typical recording of the laser power as function of time is shown in Fig. 1. The total energy of a laser pulse being monitored by a calibrated pyroelectric Joule meter was varied by changing the laser discharge voltage. As the 


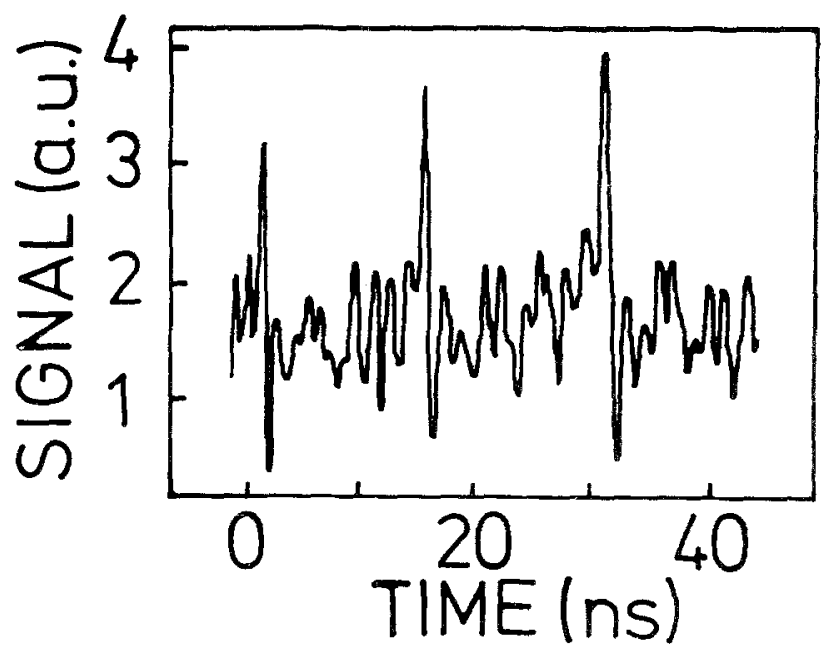

Figure 1. Shape of the high pressure $\mathrm{CO}_{2}$ Iaser pulses detected by photoconductivity of a GaPLED at $4.2 \mathrm{~K}$ for $20 \mathrm{~V}$ bias voltage, $10.6 \mu \mathrm{m}$ excitation wavelength, and $200 \mathrm{~kW} / \mathrm{cm}^{2}$ peak intensity.

shape of the pulse train did not depend on the discharge voltage the peak power and, thus the peak intensity in the sample after correcting for reflexion losses at the surface could be determined. Usually forward bias voltages up to the order of $100 \mathrm{~V}$ may be applied to GaP-LEDs at low temperatures yielding a very large photoconductive gain. This is due to the fact that below a critical forward bias voltage the electrodes of the diode are blocked by space charges captured in deep recombinative centers $[1,2]$. In the present experiment the bias voltage was limited to $10 \mathrm{~V}$ at $4.2 \mathrm{~K}$ because the diode contacts were destroyed by the large photocurrent above this voltage. In the case of $77 \mathrm{~K}$ temperature the measurements were carried out below $5 \mathrm{~V}$ bias because the dark current rapidly increased above this voltage and strongly reduced the signal-to-noise ratio. 
Results and Discussion

In Fig. 2 the short circuit photocurrent is plotted as a function of intensity $I$ measured at liquid helium and 1iquid nitrogen temperature, respectively. In both cases the photosignal clearly saturates. The observed dependence of the photocurrent on the incident intensity can be described by the phenomenological equation

$$
I_{p h}=R P\left(1+I / I_{S}\right)^{-1}
$$

where $R$ is the current responsivity, $P$ the power incident on the diode corresponding to the intensity $I$ in the diode and $I_{S}$ the saturation intensity. By fitting the experimental results to this relation, shown in Fig. 2 by the full lines, we find $\mathrm{R} \simeq 2 \cdot 10^{-4} \mathrm{~A} / \mathrm{W}$ and $2 \cdot 10^{-5} \mathrm{~A} / \mathrm{W}$ and $\mathrm{I}_{\mathrm{S}}=$ $670 \mathrm{~kW} / \mathrm{cm}^{2}$ and $180 \mathrm{~kW} / \mathrm{cm}^{2}$ for $4.2 \mathrm{~K}$ (10 V bias voltage) and $77 \mathrm{~K}(4.5 \mathrm{~V})$, respectively. These extremely large saturation intensities imply correspondingly short lifetimes of infrared excited free carriers. Besides the free hole-to-bound-donor interband recombination process, which has been proved to be fast, the capture of free carriers by ionized shallow impurities must play an essential role. The inverse lifetime $\tau^{-1}$ due to the latter process is proportional to the compensation ratio of the material. Because the p-side of the diode was prepared by counter doping Te donors diffusing from the substrate into the deposited layer during epitaxial growth, the compensation of the $p$ side is larger than that of the $n$ side. Thus the lifetime of free holes is expected to be much smaller than that of free electrons. Therefore we may assume that the photocurrent due to high power excitation is due to free holes only.

In order to obtain a physical understanding of the saturation intensity we apply a rate equation model including the acceptor ground state and the valence band. The optical absorption cross section $\sigma$ of $\mathrm{Zn}$ acceptors at 10.6 $\mu \mathrm{m}$ wavelength was evaluated from the absorption coefficient of $\mathrm{Zn}$ doped GaP [8]. Using the obtained value $\sigma=5 \cdot 10^{-16} \mathrm{~cm}^{2}$ we find an optical excitation rate per neutral acceptor of $\sigma I / \hbar \omega=5 \cdot 10^{9} \mathrm{~s}^{-1}$ for an intensity $I=200 \mathrm{~kW} / \mathrm{cm}^{2}$ where $\hbar \omega$ is the infrared photon energy. As the relaxation of nonequilibrium carriers in a band occurs on a time scale in the order of picoseconds [9] the infrared excitation of holes into the valence band is much 

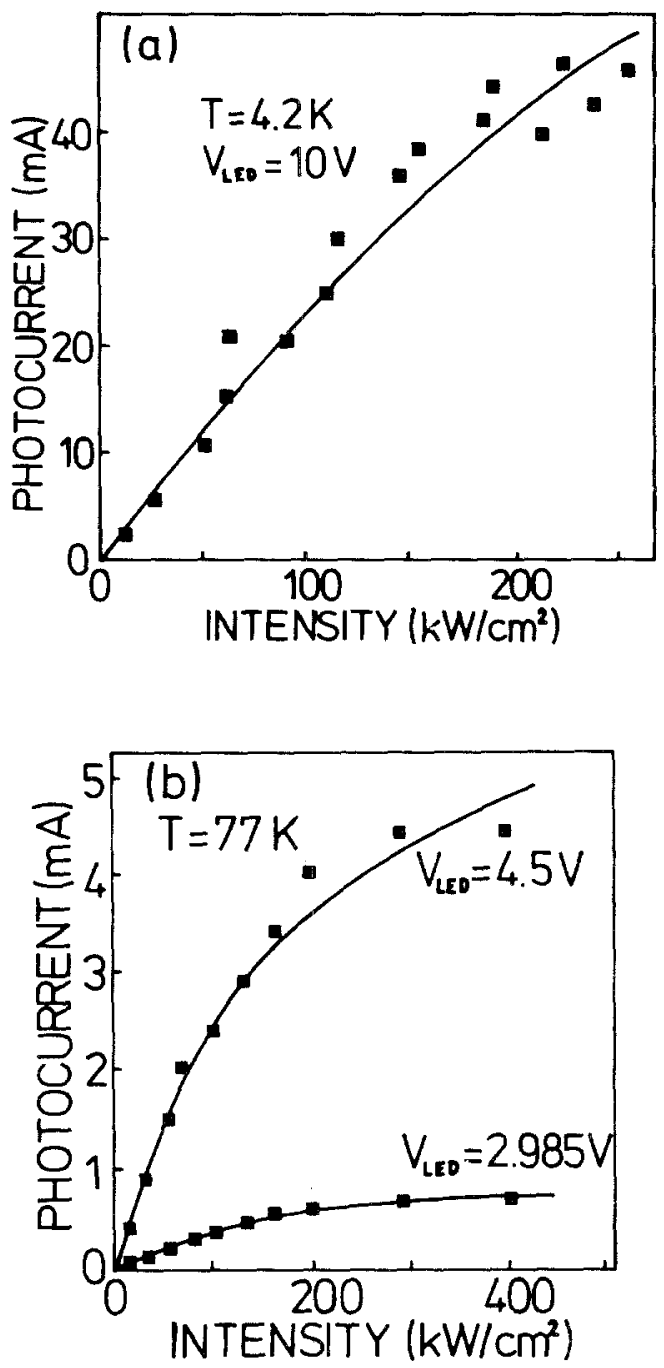

Figure 2. Peak photocurrent of the GaP diode as a function of the peak intensity of the high pressure $\mathrm{CO}_{2}$ laser at $4.2 \mathrm{~K}$. The solid lines represent a fit according to Er.1.

(a) Bias voltage: $10 \mathrm{~V}$; temperature: $4.2 \mathrm{~K}$;

(b) Bias voltage: $3 \mathrm{~V}$ and $4.5 \mathrm{~V}$; temperature $77 \mathrm{~K}$. 
slower than the relaxation. Therefore recombination of holes into ionized acceptors by stimulated emission may be neglected. In this case the rate equation determining the infrared excited concentration of holes $\Delta h$ is simply

$$
\frac{\mathrm{d}(\Delta \mathrm{h})}{\mathrm{dt}}=\left(\mathrm{N}_{\mathrm{A}^{\mathrm{O}}}-\Delta \mathrm{h}\right) \sigma \mathrm{I} / \hbar \omega-\frac{\Delta \mathrm{h}}{\tau}
$$

where $\mathrm{N}_{\mathrm{A}^{\mathrm{O}}}$ is the density of neutral acceptors. As the duration of the infrared laser pulses was much longer than the expected lifetime $\tau$, steady state conditions $\left(\frac{\mathrm{d} \Delta \mathrm{h}}{\mathrm{dt}}=0\right)$ may be presumed yielding

$$
\Delta h=\mathrm{N}_{A^{0}} \sigma \tau I / \hbar \omega\left(1+\frac{I}{I_{S}}\right)^{-1}
$$

with $I_{S}=\hbar \omega / \sigma \tau$. From this relation we find $\tau=60$ ps and $\tau=200 \mathrm{ps}$ being independent of the bias voltage up to $10 \mathrm{~V}$ and $4.5 \mathrm{~V}$ for $4.2 \mathrm{~K}$ and $77 \mathrm{~K}$, respectively. These extremely short 1 ifetimes cannot be explained by radiative free-holeto-bound donor recombination, whose time constant was estimated to be about 2 ns [4], or by any other interband transition. Thus these results concerning $\tau$ additionally justify the simple model leading to $\mathrm{Eq}$. 1. The inverse lifetime may be expressed by $\tau^{-l}=\sigma_{c}\langle v\rangle N_{A}$ where $\sigma_{c}$ is capture cross section of ionized acceptors, <v> the average velocity of free holes, and $\mathrm{N}_{\mathrm{A}^{-}}$the concentration of ionized acceptors.

In compensated material at low temperature $(4.2 \mathrm{~K})$ the concentration of ionized acceptors is equal to the sum of the concentrations of the donors $\mathrm{N}_{D}$ and the free infrared excited holes $\Delta h$

$$
\mathrm{N}_{\mathrm{A}^{-}}=\mathrm{N}_{\mathrm{D}}+\Delta \mathrm{h}
$$

where $\mathrm{N}_{\mathrm{D}}$ is in the order of magnitude $\mathrm{N}_{\mathrm{A}^{\mathrm{O}}} / 100$ to $\mathrm{N}_{\mathrm{A}^{\mathrm{O}}} / 10$ and $\Delta \mathrm{h}<\mathrm{N}_{\mathrm{D}} / 10$. for the highest intensities employed. Therefore we get $\mathrm{N}_{\mathrm{A}^{-}} \simeq \mathrm{N}_{\mathrm{D}}$ at low temperature and even when all acceptors are thermally ionized at higher temperatures $\mathrm{N}_{\mathrm{A}^{-}}$cannot exceed this value by more than 2 orders of magnitude. The capture cross section $\sigma_{c}$ decreases, however, very strongly with increasing temperature, approximately like $\sigma_{\mathrm{c}} \sim 1 / \mathrm{T}^{2}[10]$ and $\langle\mathrm{v}\rangle$ increases with temperature like 
$\langle v\rangle \sim \sqrt{\mathrm{T}}$ and with injection of charge carriers. As the electric field gradient in the diode is not known accurately at low temperatures, $\langle v\rangle$ cannot be evaluated exactly. The product $\sigma_{\mathrm{C}}\langle\mathrm{v}\rangle$ will nevertheless approximately vary like $\mathrm{T}^{-3 / 2}$ and therefore decrease from $4.2 \mathrm{~K}$ to $77 \mathrm{~K}$ by a factor of about 80 . The time constants determined from the saturation intensity at the two different temperatures and thus explainable if $\mathrm{N}_{\mathrm{D}} \simeq \mathrm{N}_{\mathrm{AO}} / 25$.

It is obvious that this mechanism can have some influence on other recombination processes; especially the decay of free-to-bound and donor acceptor pair luminescence will be affected.

\section{Conclusion}

We have shown that in GaP light emitting diodes used as extrinsic photoconductive detectors at low temperatures saturation occurs at intensities of several $100 \mathrm{~kW} / \mathrm{cm}^{2}$. From the saturation intensities the lifetime of infrared excited holes in the valence band could by derived to be of the order of $100 \mathrm{ps}$. It is expected that the time constant of photoconductivity is of the same order of magnitude. Thus GaP diodes represent very fast infrared detectors having a large dynamical range.

\section{Acknowledgements}

We are indebted to Dr. N. Stath and J. Attenhauser (Siemens $A G$, Regensburg) for providing the diode samples and helpful discussions. We also thank $U$. Werling for experimental support with the high pressure $\mathrm{CO}_{2}$ laser. Financial support by the Deutsche Forschungsgemeinschaft is gratefully acknowledged.

\section{References}

1. W. Eisfeld, U. Werling, and W. Prett1, App1. Phys. Lett. 42, 276 (1983).

2. K. Moser, S. Wahl, W. Eisfeld, and W. Prett1, J. App1. Phys., 57, 5438 (1985). 
3. K. Moser, W. Eisfeld, U. Werling, S. Wahl, and W. Prett1, App1. Phys. Lett. 45, 711 (1984).

4. K. Moser, W. Eisfe1d, and W. Prett1, Infrared Phys. 4, 659 (1985).

5. K. Moser, W. Eisfeld, W. Penzenstadler, and W. Prett1, J. Phys. D, to be published.

6. Wan Chong-Yi, U. Werling, and K.F. Renk, J. App1. Phys. 57, 990 (1985).

7. U. Werling, Wan Chong-Yi, and K.F. Renk, Intern. J. of Infrared and Millimeter Waves 6 , 449 (1985).

8. A.A. Kopylov, A.N. Pikhtin, Solid State Commun. 26, 735 (1977).

9. Jagdeep Shah, Solid State Electr. 21, 43 (1978).

10. P.R. Bratt, in "Semiconductors and Semimetals", ed. by R.K. Willardson and A.C. Beer, Academic Press, New York 1977, vol. 12, p. 39. 